\title{
MINERAL COMPOSITION OF ESTONIAN OIL SHALE SEMI-COKE SEDIMENTS
}

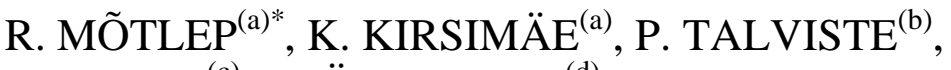 \\ E. PUURA ${ }^{(\mathrm{c})}$, J. JÜRGENSON ${ }^{(\mathrm{d})}$ \\ (a) Institute of Geology \\ University of Tartu \\ Vanemuise 46, 51014 Tartu, Estonia \\ (b) IPT Projektijuhtimine OÜ \\ Kopli 96-1, 10416 Tallinn, Estonia \\ (c) Institute of Technology \\ University of Tartu \\ Nooruse 1, 50411 Tartu, Estonia \\ (d) AS Viru Keemia Grupp \\ Järveküla tee 14, 30328 Kohtla-Järve, Estonia
}

\begin{abstract}
Semi-coke is a solid waste material left after oil shale retorting in oil shale chemical industry. During more than 80 years of operation more than 110 million tonnes of semicoke have been deposited at retorting plants in Estonia but so far very little is known about the mineral composition of this waste. This paper presents new data on mineral composition of semi-coke and its spatial variation in waste heaps. The composition of semi-coke reflects the changes of mineral matter during the retorting process. The changes with the terrigenous and carbonate matter is almost negligible during the main phase of retorting, only dehydration and partial transformation of clay minerals and decomposition of sulfur compounds occur. At the final step of retorting $\left(900-1000^{\circ} \mathrm{C}\right)$ a slag-like material forms, which consists of amorphous and Ca-silicate phases.

The deposition and subsequent hydration causes the change in the composition of semi-coke. The most notable change is the formation of ettringite. The composition of mineral matter in semi-coke waste heap is relatively uniform. The variations are probably due to both physical and chemical separation during deposition of the sediment and to different diagenetic/hydration processes.
\end{abstract}

\footnotetext{
*Corresponding author: e-mail riho.motlep@ut.ee
} 


\section{Introduction}

Estonian kukersite is the largest oil shale resource in the world used industrially today [1]. The majority of mined oil shale is utilized for production of electricity and heat. About $20 \%$ of all of oil shale extracted annually is used for retorting to produce shale oil and shale gas, after which large amounts of solid retort residue - semi-coke - are left over. Production of one tonne of shale oil is accompanied by almost 3 tonnes of semi-coke waste. Two different retorting processes are used in Estonian oil shale chemical industry [2]. Their net oil yield efficiency is $11-17.5 \%$, and the amount of organics left in semi-coke is considerably higher (up to 16\%) as compared to coal- or oil-based chemical industry. Residual organics in semicoke and leachates from it contain phenols, PAHs (polycyclic aromatic hydrocarbons) and oil products that are potential pollutants with harmful environmental effect. Retorting process of oil shale is also accompanied with generation of condensate/pyrogenous water contaminated with organics, fuses and several other toxic products. Another concern is high alkalinity of semi-coke leachates [3].

Since the year 1921 when oil shale retorting started in Estonia, about 110 million tonnes of semi-coke have been deposited at retorting plants. The three largest installations are at Eesti Power Plant (AS Narva EJ Õlitehas), Kohtla-Järve (Viru Keemia Grupp AS) and Kiviõli (Kiviõli Keemiatööstuse $O \ddot{U})$. Finding ways for semi-coke utilization is very important in light of its environmental effects, and increasing environmental taxes may render the shale oil production economically unprofitable. For decades, starting from the '50s semi-coke was mostly used in production of mineral wool and to a smaller extent in cement and compost production. Total mineral wool production between 1959-1985 was $\sim 5.3$ million $\mathrm{m}^{3}$, estimated share of total semi-coke in feed was 0.83 million tonnes [4]. Today, small amount of semicoke is used as an ingredient of composts in mixture with pig slurry and peat and for manufacturing of rock wool. A minor amount of semi-coke (10 $000 \mathrm{t}$ annually) has been used for cement production in Kunda Nordic Cement Ltd. However, utilization of semi-coke in this way and in such amounts only mitigates the situation but does not resolve the problem. Several methods for complete utilization of semi-coke have been proposed. Kuusik et al., [5] carried out an extensive study to find the most suitable energy and costeffective methods for utilization of semi-coke as a fuel. They proposed combustion of semi-coke in specially designed circulating fluidized bed (CFB) boilers at the place of waste formation and as an additive to a fuel(s) used in cement clinker production.

The potential environmental hazardousness and possibilities of semi-coke reusability depends on the content of its organic component and on the composition of mineral matter. Although different aspects of Estonian oil shale, its geology, composition and use are well studied (e.g. [6, 7]), to our best knowledge there is no comprehensive study on the semi-coke mineral- 
ogy and the mineral composition of the semi-coke and its deposits is virtually unknown.

The objectives of this paper is to study, first, the formation and stability of the mineral matter during partial thermal decomposition and subsequent reactions during the retorting process, second, transformation processes during hydration of semi-coke under atmospheric conditions in the semicoke waste heaps and third, the composition and spatial variation of semicoke sediments in waste heaps.

\section{Composition of oil shale and retorting process}

Oil shales are fine-grained sedimentary rocks that contain relatively large amounts (10-65\%) of organic matter (kerogen) from which significant amounts of shale oil and combustible gas can be extracted by destructive distillation. Oil shales are found world-wide in all types of marine, lacustrine or terrestrial sedimentary rocks of Cambrian to Neogene age. Organic rich calcareous sediment - kukersite - found in sediments of Lower to Upper Ordovician age in Estonia and north-western Russia is a marine oil shale [8]. The organic matter of kukersite is composed mostly of kerogen with few percent of bitumen [9]. It consists almost entirely of accumulations of discrete bodies, telalginite, derived from a colonial microorganism Gloeocapsomorpha prisca $[9,10]$. However, Kattai et al. [6] point out that the kukersite kerogen is more similar to lacustrine oil shales and sapropelic coals than to the kerogen of typical marine oil shales.

Besides organic matter, kukersite contains significant amounts of carbonaceous and terrigenous matter. The last two constitute kukersite's mineral matter. Oil shale consists of 10 to $65 \%$ of organics, $20-70 \%$ of carbonate minerals and 15-60\% of terrigenous minerals (quartz, feldspars and clay minerals), on dry weight basis (Kattai et al. [6]. The major components of organic matrix are phenolic moieties with linear alkyl sidechains [3]. The average content of carbon in the organic matter of kukersite is low $(76.7 \%)$ with sulfur content of $1.6 \%$. The moisture content is $9-12 \%$, and calorific value $-8-10 \mathrm{MJ} / \mathrm{kg}$, which is lower than that of the other fuels (as an example the average coal $-22.5 \mathrm{MJ} / \mathrm{kg}$, natural gas $-33.5 \mathrm{MJ} / \mathrm{m}^{3}$, peat briquette $-16.5 \mathrm{MJ} / \mathrm{kg}$ ) [11]. According to Utsal [12], the average mineral composition of kukersite mineral part is dominated by carbonate minerals (50-60\%) including mostly calcite (40-45\%) and dolomite (5-15\%). The content of clay minerals, quartz and feldspars (K-fledspar) vary around 20,11 and 7\%, respectively. The mineral matter of kukersite contains also $5-10 \%$ of pyrite.

During oil shale retorting, the shale is heated in the absence of oxygen to the temperature at which kerogen is decomposed or pyrolysed into gas, condensable oil, and solid residue. The inorganic mineral matrix of the shale is retained in the form of spent shale [9]. Thermal destruction of kukersite 
begins at $170-180{ }^{\circ} \mathrm{C}$, and pyrogenous water appears at $270-290{ }^{\circ} \mathrm{C}$. At the temperature $350-400{ }^{\circ} \mathrm{C}$, the organic substance of kukersite is converted into semi-liquid state, called thermobitumen [13]. The rate of kerogen (thermobitumen) decomposition is the highest at retort temperature of $480-520{ }^{\circ} \mathrm{C}$, at which kerogen converts into three organic fractions: oil, gas and residual carbon. The gases and vapours leaving the retort are cooled to condense the oils and process water. Oil shale must pass the temperatures of thermobitumen formation and coking at a relatively high speed to avoid cracking and secondary pyrolysis of oil [9]. Over the past two decades, two main methods have been used in producing shale oil, namely: the "Kiviter" process and "Galoter" (Solid Heat Carrier, SHC) process. The Kiviter process is conducted in a vertical gas generator (GGS) (retort throughput $1000 \mathrm{t}$ per day), which is internally heated by combustion of coke residue and non-condensable shale gas. Operation of the Kiviter retort is continuous whereas the heat is provided by the rising gases, supplemented by recycle gas, burned in the heat carrier preparation chamber. Additional recycled gas and the air admitted to the chambers near $900{ }^{\circ} \mathrm{C}$ point heat the shale residue to burn off the coke at the last stage of retorting [14]. The Galoter retort uses spent shale as a heat carrier. The process is based on introducing dried oil shale $(<2.5 \mathrm{~cm}$ particle size) into an aerofountain drier where it is mixed with hot $\left(590-650{ }^{\circ} \mathrm{C}\right)$ shale ash produced by combustion of oil shale semicoke (at $740-810^{\circ} \mathrm{C}$ at oxygen deficiency) [2].

Total of 111 million tonnes of semi-coke has deposited in heaps covering more than 200 ha. Most of it is deposited at Kohtla-Järve (83.22 Mt), Kiviõli (19.2 Mt) and Narva (4.34 Mt). Other minor locations Vanamõisa, KohtlaNõmme and Sillamäe hold in total of $4.56 \mathrm{Mt}$ semi-coke [4].

\section{Material and methods}

Studied semi-coke samples were collected at Viru Keemia Grupp AS semicoke waste deposit plateau in Kohtla-Järve (Fig. 1). The plateau refers to the central flat area on the waste heap where semi-coke is deposited today. Mineralogical analyses were performed on fresh semi-coke, waste plateau surface sediments and on samples from six drillcores. Altogether 47 samples were investigated. The mineral composition of semi-coke was determined by means of X-ray powder diffractometry (XRD). XRD analyses were carried out on (1) unhydrated (fresh) semi-coke; (2) recently deposited semi-coke exposed for two weeks to atmospheric conditions and (3) drillcore samples from depth range $0.8-14.7 \mathrm{~m}$ at the semi-coke waste heap (Fig. 1). To avoid additional hydration and carbonation all samples were stored airtight in a refrigerator prior to analysis. To minimize any hydration and oxidation effects on fresh semi-coke, the samples were frozen instantly in liquid nitrogen and sublimated in vacuum drier prior to analyses. XRD preparations made from samples were measured within 4 hours after the preparation was 
made. The waste plateau samples were dried at $105{ }^{\circ} \mathrm{C}$ for $24 \mathrm{~h}$ prior to XRD preparation.

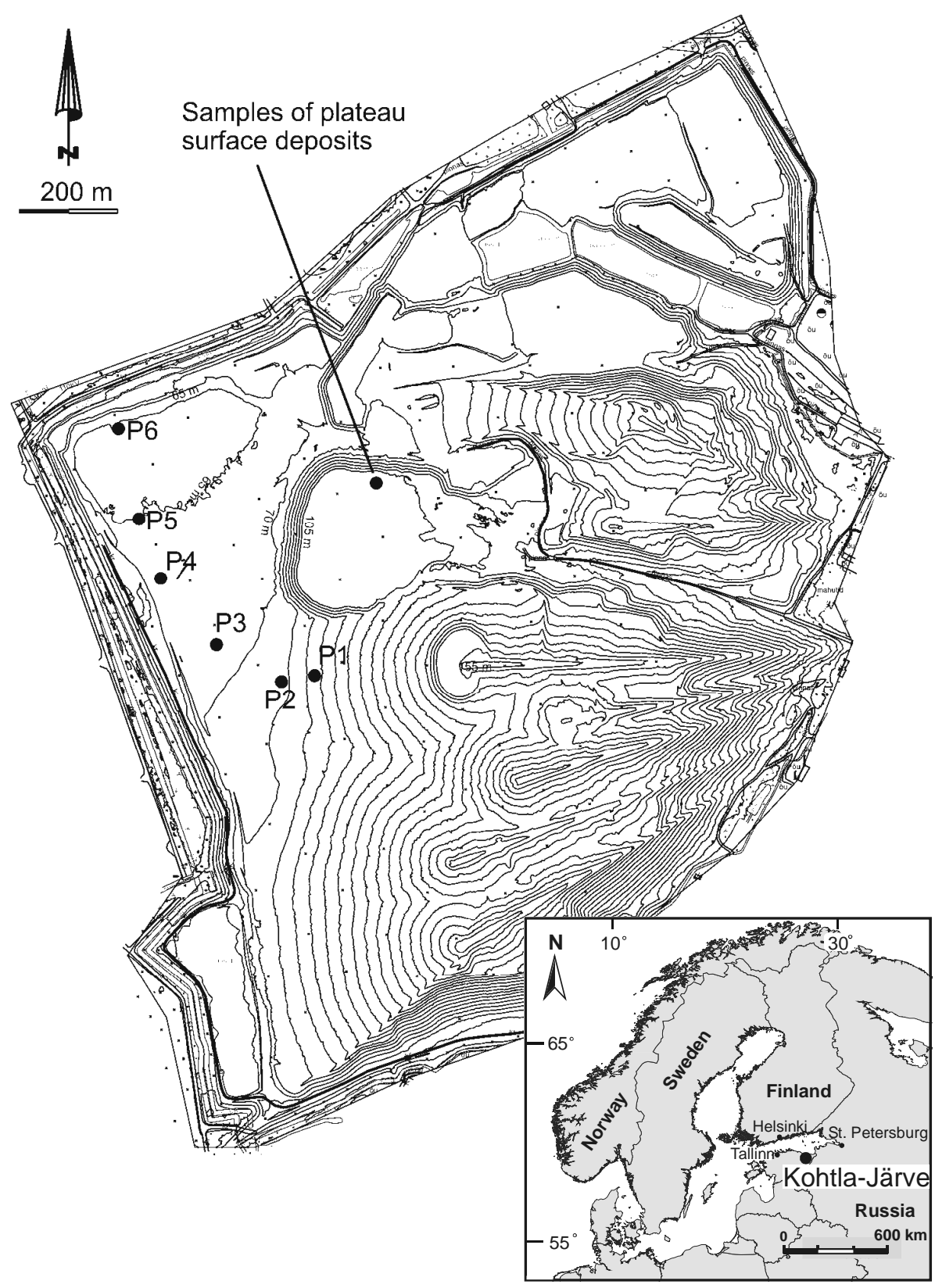

Fig. 1. Scheme of semi-coke waste heaps, location of studied drillcores and the sampling point at the surface (Courtesy of Viru Keemia Grupp AS, topography by EOMap, 2004). 
The mineral composition of studied samples was determined using Rietveld technique of X-ray diffraction analysis. Grinded and homogenized unoriented powder samples were prepared on Al-sample holders and exposed to $\mathrm{Cu} K \alpha$ radiation on Dron-3M diffractometer. Digitally registered diffractograms were measured at the interval between $2-50^{\circ} 2 \theta$, with step $0.03{ }^{\circ} 2 \theta$ and counting time $3-5$ seconds. Quantitative mineral content was measured by Rietveld analyze based program SIROQUANT-2.5 ${ }^{\mathrm{TM}}[15]$. The full profile XRD pattern Rietveld method takes into account integrated intensities of the particular diffractogram peaks and compares natural and artificial mixtures along the whole length of the diffractogram, providing thus considerably more information for mineral quantification [16]. Allowance was made in the SIROQUANT analysis for preferred orientation in several minerals, such as carbonates, whereas a match was obtained for the key peak positions, the distribution of peak heights was inconsistent with the standard mineral pattern. The standard patterns for such minerals were refined by SIROQUANT using computations based on the March-Dollase Function preferred orientation correction [17] and the mineral percentages adjusted accordingly [16]. For some of the minerals indicated by SIROQUANT to be present in low concentrations $(<1 \%)$ the estimated error is of magnitude similar to, if not greater than, the actual determination. In tabulations of the present work these minerals are shown as traces (marked in table as tr.), and they are not taken into account when the remaining components were normalized to $100 \%$. Micromorphology and spatial relationships of secondary mineralization were carried out using scanning electron microscope (SEM) Zeiss DSM 940. SEM preparations were coated with conductive gold coating during preparation.

\section{Results}

The mineral composition and representative XRD patterns of studied samples are shown in Tables 1, 2 and Fig. 2.

Table 1. Average mineral composition of fresh semi-coke and the same material hydrated sampled after two weeks of hydration under ambient conditions, wt\%; tr. - traces

\begin{tabular}{|c|c|c|c|c|c|c|c|c|c|c|c|c|c|c|}
\hline $\begin{array}{l}\frac{\vec{J}}{\bar{J}} \\
\frac{\vec{J}}{\Sigma}\end{array}$ & 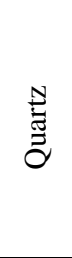 & 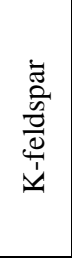 & 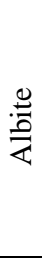 & 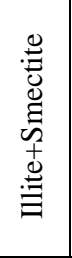 & 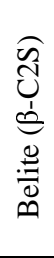 & 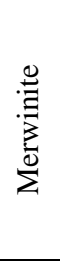 & తֶ & $\begin{array}{l}0 \\
\frac{0}{0} \\
\frac{0}{0} \\
0\end{array}$ & 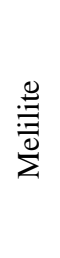 & $\frac{\mathscr{n}}{\frac{\pi}{\pi}}$ & $\begin{array}{l}\stackrel{\mathscr{\Xi}}{\frac{0}{0}} \\
\frac{0}{\circ}\end{array}$ & 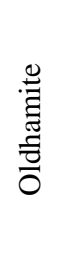 & 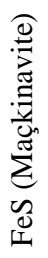 & 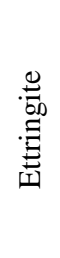 \\
\hline Fresh & 13.6 & 18.6 & tr. & 15.3 & 5.4 & 2.8 & tr. & 2.0 & 9.5 & 25.5 & 4.3 & 3.0 & tr. & \\
\hline Hydrated & 11.4 & 11.2 & tr. & 14.3 & 6.6 & 4.0 & tr. & 1.5 & 5.4 & 29.3 & 3.1 & & & 13.2 \\
\hline
\end{tabular}




\begin{tabular}{|c|c|c|c|c|c|c|}
\hline$\mp$ & $\begin{array}{ll}\hat{m} & \bar{m} \\
0 & 0\end{array}$ & & $\overbrace{0}^{\circ}$ & & & \\
\hline әseppinad & $\stackrel{\circ}{\circ}$ & & $\stackrel{\circ}{+}$ & & & \\
\hline$\mp$ & & & 舟 & & & \\
\hline ә॥soder & & & $\stackrel{m}{N}$ & & & \\
\hline$\neq$ & 岕 & 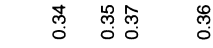 & & 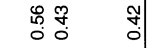 & รั & F. \\
\hline unsd $K \emptyset$ & 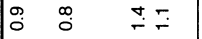 & $\stackrel{\infty}{=} \stackrel{\varphi}{=}=\stackrel{\circ}{=}$ & $=$ & 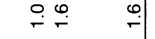 & $\stackrel{\circ}{\mathrm{N}}$ & $\stackrel{0}{\circ} \stackrel{9}{0}=$ \\
\hline$\mp$ & 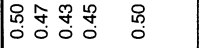 & & & & 然 & \\
\hline әци!мдәพ & 饨 & & & & $\stackrel{N}{N} \bar{N}$ & \\
\hline 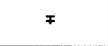 & & 兽 & ?ุ' & 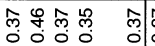 & 畣兽 & \\
\hline $\begin{array}{l}\text { oylun|es } \\
\text {-odp } K_{H}\end{array}$ & $\doteq \doteq \doteq \doteq \doteq \doteq$ & $\doteq$ & $\stackrel{\infty}{-}$ & 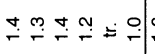 & $\stackrel{r}{=}$ & \\
\hline$F$ & 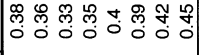 & 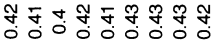 & $\mid$ & $\mid$ & 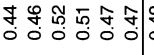 & 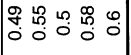 \\
\hline คี!!!!อพ & 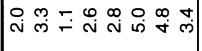 & 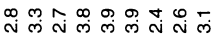 & 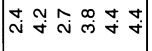 & 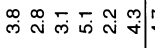 & 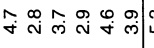 & 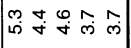 \\
\hline$\mp$ & 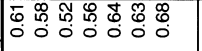 & 芯 & 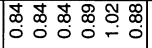 & 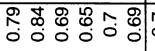 & 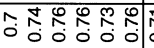 & 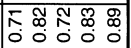 \\
\hline 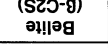 & 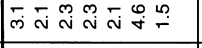 & 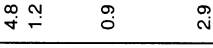 & 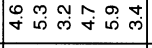 & 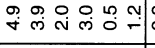 & 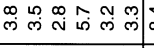 & 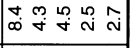 \\
\hline$\mp$ & 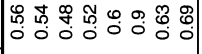 & 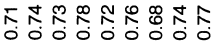 & 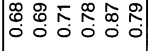 & $\mid$ & $\begin{array}{lll} \\
0 \\
0\end{array}$ & $\mid$ \\
\hline อมбธบเมี & N & 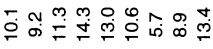 & 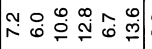 & 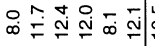 & 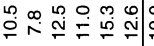 & 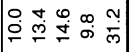 \\
\hline$\mp$ & 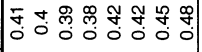 & 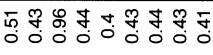 & $\mid$ & 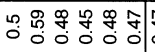 & fै & 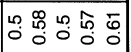 \\
\hline ә!ㅐ임ㅁ & 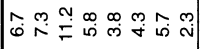 & 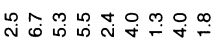 & $\mid$ & 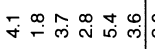 & 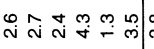 & 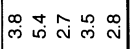 \\
\hline$\mp$ & 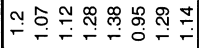 & 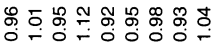 & 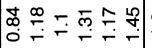 & 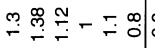 & 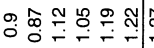 & 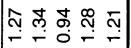 \\
\hline ә낑 & 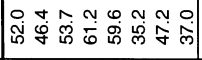 & $\begin{array}{l}\infty \\
\dot{\sigma} \\
\dot{\sigma}\end{array}$ & 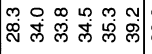 & $\begin{array}{l}\infty \\
0\end{array}$ & 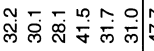 & 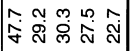 \\
\hline$\mp$ & 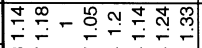 & بَ & 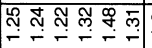 & بִ & 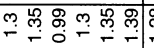 & 萬只 \\
\hline 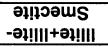 & 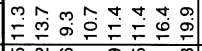 & 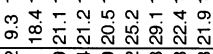 & 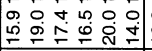 & 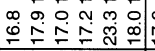 & 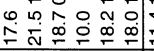 & 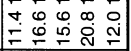 \\
\hline$\mp$ & 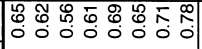 & 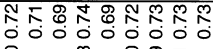 & 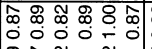 & 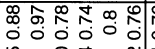 & 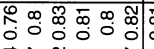 & 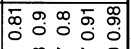 \\
\hline 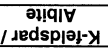 & 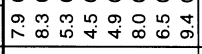 & 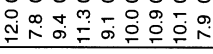 & 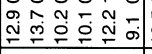 & 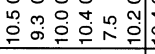 & 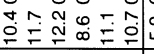 & 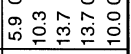 \\
\hline$\mp$ & m్ & 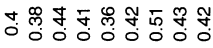 & $\mid$ & 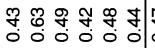 & 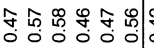 & 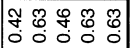 \\
\hline zueno & 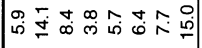 & 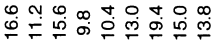 & 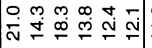 & $\mid$ & 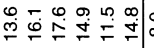 & $\mid \begin{array}{l}0 \\
0\end{array}$ \\
\hline 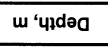 & 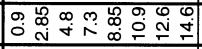 & 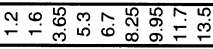 & \begin{tabular}{|lll} 
\\
\end{tabular} & 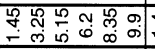 & 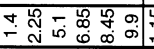 & 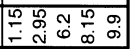 \\
\hline 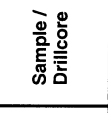 & 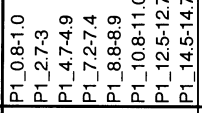 & 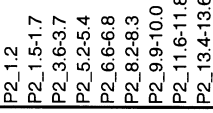 & & & 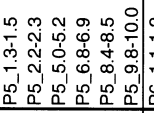 & 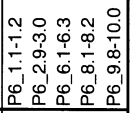 \\
\hline ou 'qe7 & 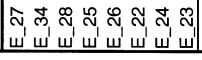 & 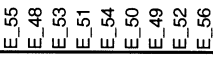 & 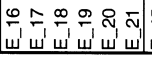 & 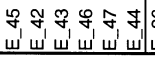 & 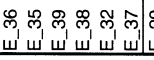 & 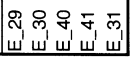 \\
\hline
\end{tabular}




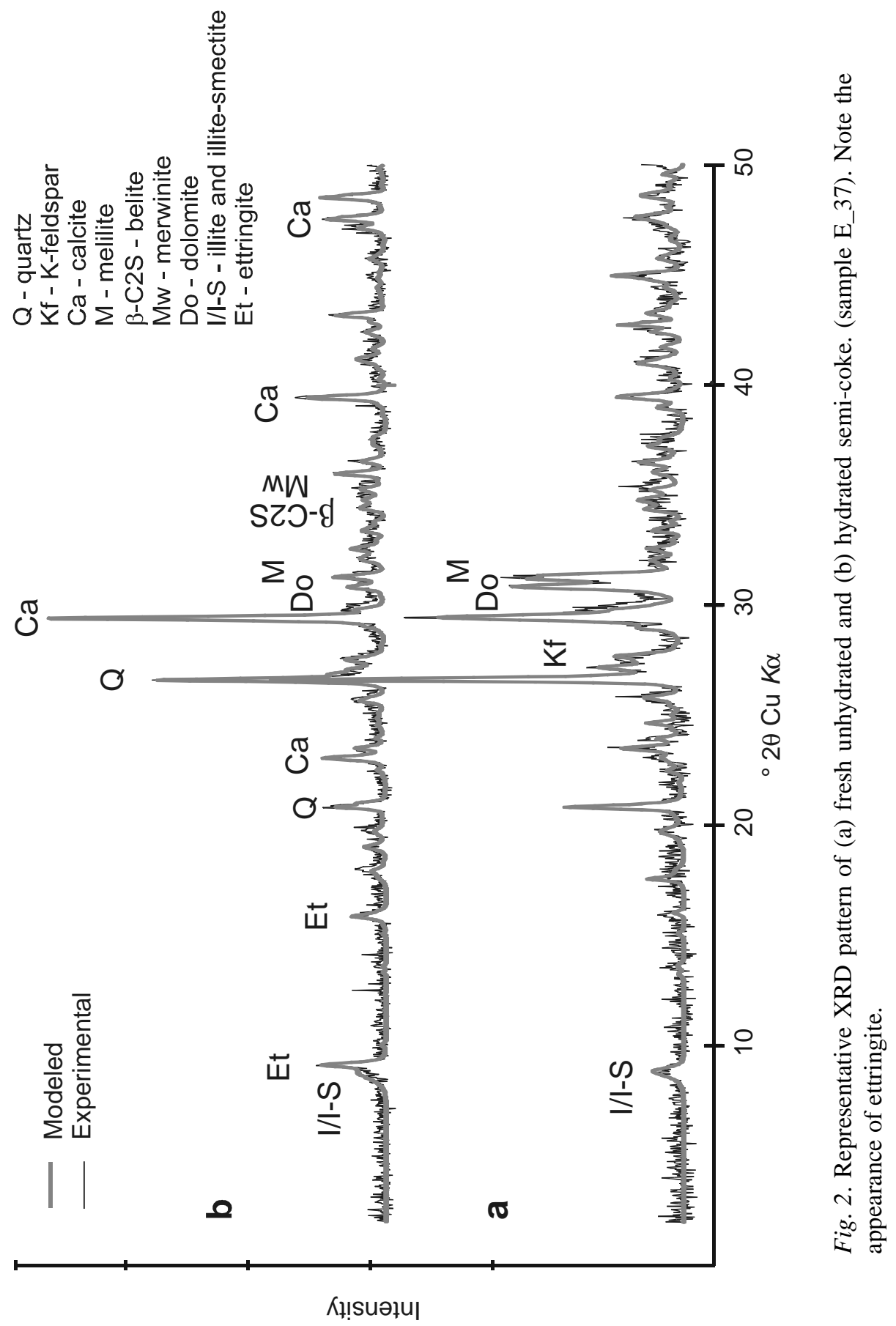




\section{XRD mineralogy}

Fresh semi-coke. The predominant phases identified by X-ray diffraction in a fresh unhydrated semi-coke (Table 1, Fig. 2) were calcite, dolomite, quartz, K-feldspar and clay minerals (mostly illite), which are typical constituents of oil shale mineral matter and were preserved or slightly changed during the retorting process. In addition to these minerals, fresh semi-coke contains secondary phases formed by partial thermal decomposition and subsequent reactions of carbonate and clay minerals - melilite $\left[(\mathrm{Ca}, \mathrm{Na})_{2}(\mathrm{Al}, \mathrm{Mg}, \mathrm{Fe})(\mathrm{Si}, \mathrm{Al})_{2} \mathrm{O}_{7}\right]$, periclase $[\mathrm{MgO}]$, clinker-minerals: belite $[\beta$-C2S $]$, merwinite $\left[\mathrm{Ca}_{3} \mathrm{Mg}(\mathrm{SiO} 4)_{2}\right]$. Also oldhamite $[\mathrm{CaS}]$ together with traces of $\mathrm{FeS}$ - mackinawite and $\mathrm{C} 3 \mathrm{~A}$ - tricalcium aluminate were identified in fresh semi-coke.

The studied samples do not contain above detecton limit (>1\%) lime $[\mathrm{CaO}]$ nor its hydration product portlandite $\left[\mathrm{Ca}(\mathrm{OH})_{2}\right]$ or anhydrite $\left[\mathrm{CaSO}_{4}\right]$, which are typical phases in oil shale combustion ashes (e.g. [18]. XRD analysis is unable to identify the amorphous phase (non-crystallised matter). However the diffuse maximum from amorphous phase scattering in measured XRD diffractogram indicates the quantity of non-crystallised matter to be as high as $10(20) \%$. Fresh semi-coke contains occasionally yellowish-green lumps of partially melted slag, which are composed of hightemperature $\mathrm{Ca}-\mathrm{Mg}$ silicates - diposide, enstatite and various clinkerminerals ( $\beta$-C2S, C3S, merwinite and traces of Ca-ferrites).

Hydrated semi-coke and plateau deposits. The most notable difference between the fresh and deposited semi-coke is the disappearance of oldhamite $[\mathrm{CaS}]$ and the occurrence of considerable amount of ettringite $\left[\mathrm{Ca}_{6} \mathrm{Al}_{2}\left(\mathrm{SO}_{4}\right)_{3}(\mathrm{OH})_{12} \cdot 26 \mathrm{H}_{2} \mathrm{O}\right]$ (Tables 1 and 2, Fig. 2). The average abundance of minerals fluctuates somewhat, which is probably due to the variations in the original deposited matter and its later alteration.

Mineral composition of semi-coke matter in plateau samples is rather uniform. However, there are certain variations evident in drillcore samples from various locations and depths. The dominant phases in all drillcore samples P1-P6 are calcite and dolomite; quartz, feldspars (K-feldspar and in trace amounts of albite), clay minerals (illite/illite-smectite); and ettringite (Fig. 3, Table 2). The content of the rest of the minerals present in studied samples (mostly clinker-minerals) are usually below 5\%. Ettringite shows considerable variation from $3.5 \%$ to $20.3 \%$, with an average of $9 \%$. An interesting feature characterizing all studied samples is a slight increase in ettringite content with increasing depth of sampling. However, the increase is not expressed very clearly and the variation is considerable (Fig. 4). Also, there is a small, but consistent increase of ettringite along the profile from the uppermost drillcore P1 to the lowermost cores P5 and P6 (Fig. 5). 

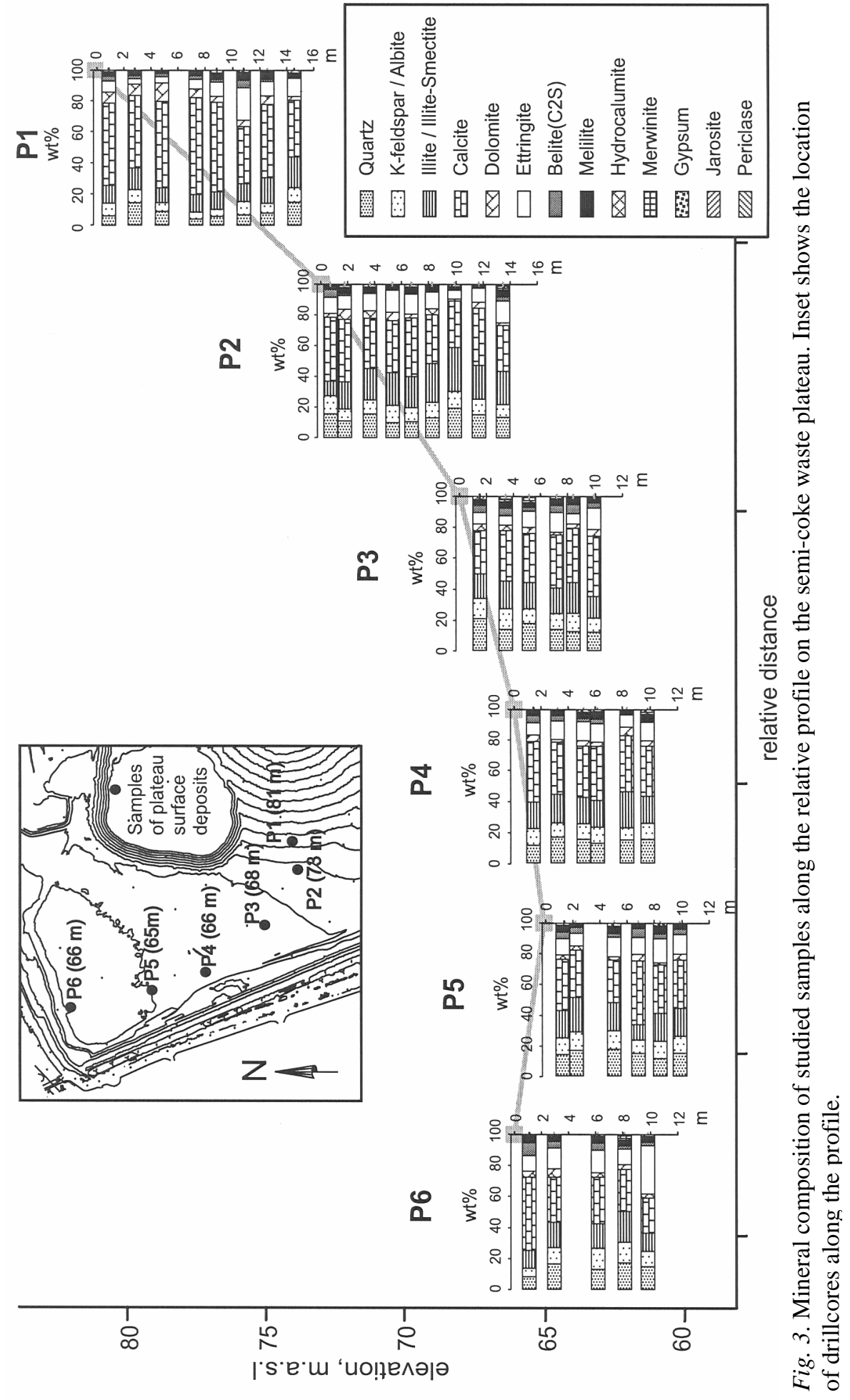

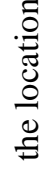



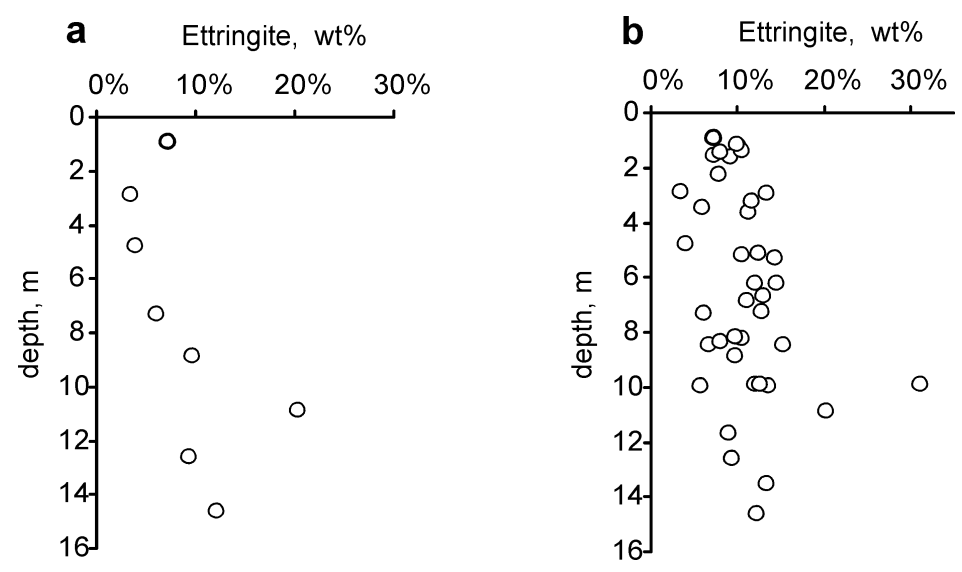

Fig. 4. Ettringite content variation with the depth. (a) - drillcore P1 and (b) - all studied drillcore samples.

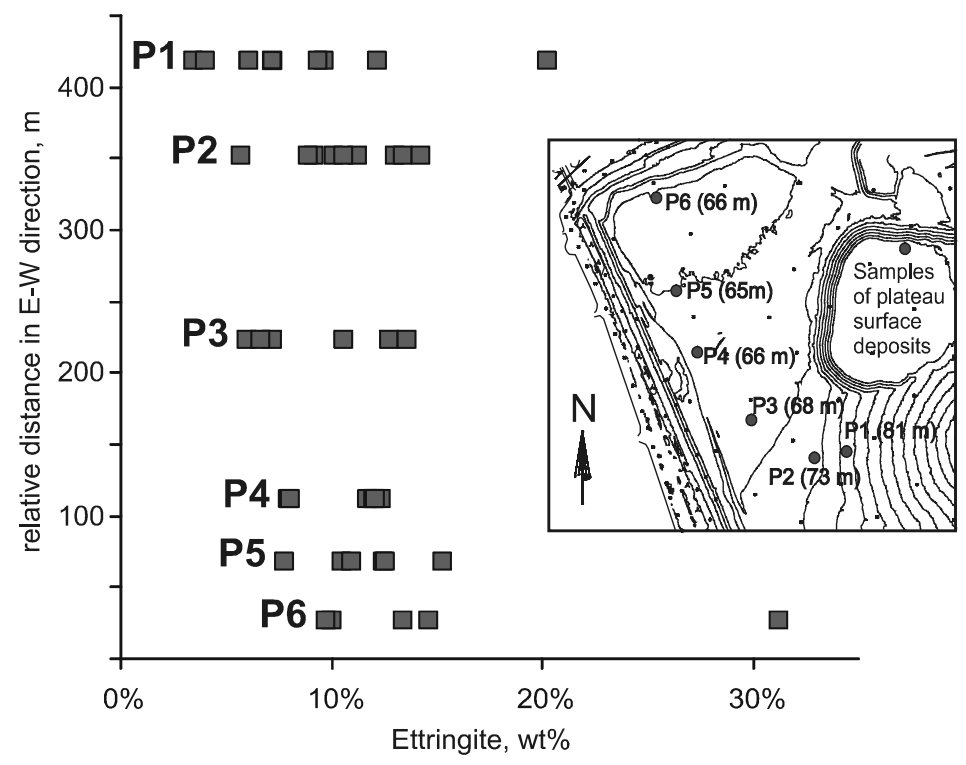

Fig. 5. Ettringite content along the P1- P6 drillcore profile. The inset shows the location and height of the drillcores.

\section{Scanning electron microscopy}

Scanning electron microscopy (SEM) studies were undertaken primarily to study the occurrence and spatial distribution of ettringite as a major secondary phase. SEM micrographs of unhydrated semi-coke show very porous material (Fig. 6a, b, c), composed of different sized particles. Secondary ettringite forms $2-10 \mu \mathrm{m}$ long prismatic crystals that precipitate and form irregular aggregates in semi-coke open pores (Fig. 6d, e). In P5 drillcore samples ettringite crystals show signs of partial dissolution (Fig. 6f). 

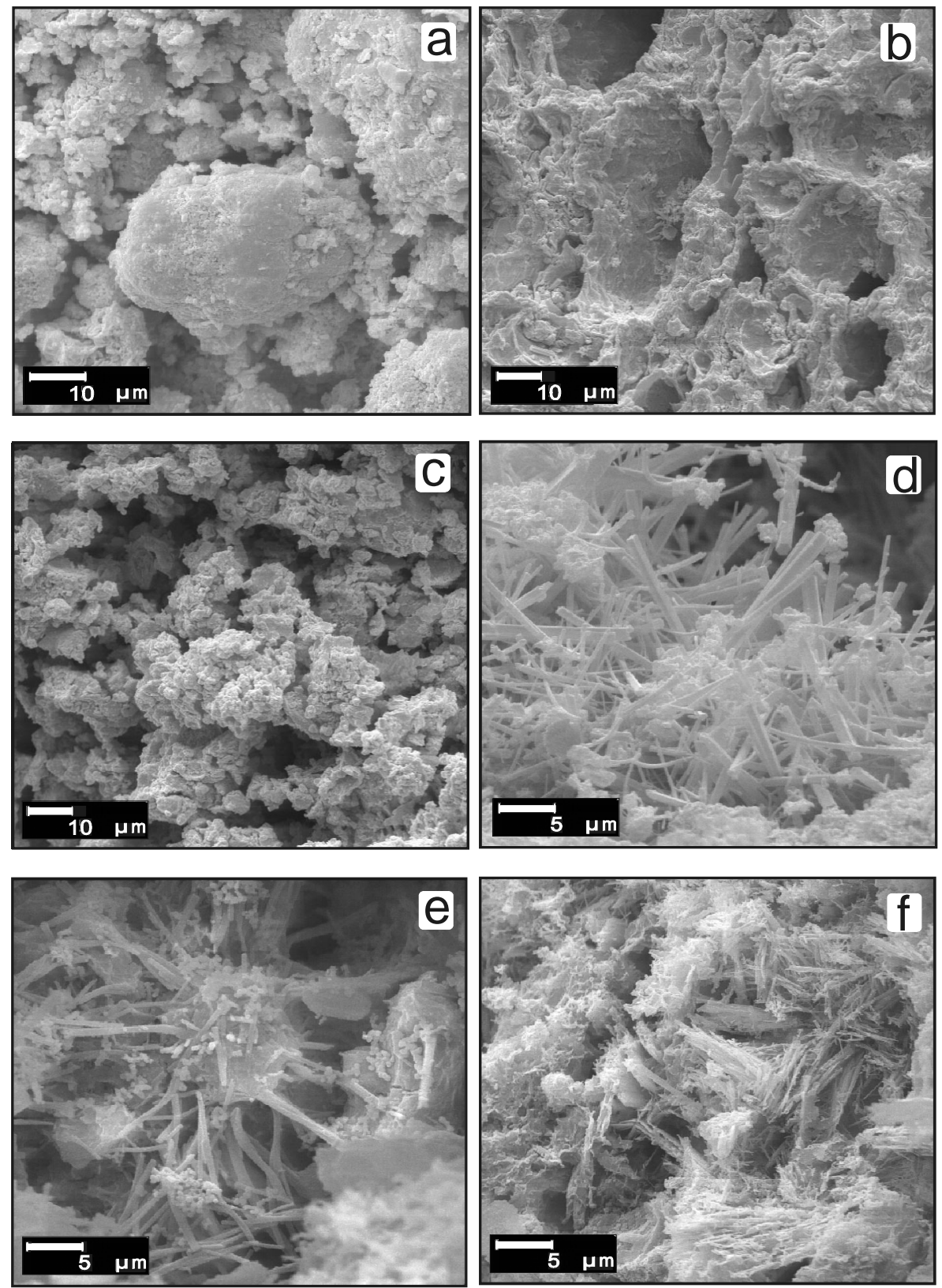

Fig. 6. SEM secondary electron (SE) images of semi-coke samples. (a) - fresh, unhydrated sample. (b) - vesicular microstructure of partially melted lumps in semicoke, note the diagenetically altered surfaces. Semi-coke is very porous material (c), where ettringite forms irregular prismatic crystallites (d, e); (f) - a micrograph of partially dissolved ettringite crystals. 


\section{Discussion}

\section{Mineral transformation in retorting process}

The solid retort residue in Kiviter process - semi-coke - is formed by the transformation of oil shale as a result of its partial thermal decomposition in two stages:

(1) semi-coking process at temperatures of $400-520{ }^{\circ} \mathrm{C}$, and

(2) short heating at $900-1000{ }^{\circ} \mathrm{C}$ during the final stage of retorting, which is primarily aimed to burn off the coke (organic) residue.

Semi-coke is a complex mixture, which contains alongside with transformed matter also pieces of macroscopically unaltered oil shale that shows only slight signs of thermal decomposition. Semi-coke occasionally contains lumps-pieces of partly altered carbonate rock together with lumps of melted slag. The changes of mineral matter during the first stage of the retorting process, which occurs at air deficiency conditions, are negligible. The terrigenous fractions of the oil shale - quartz and feldspars - remain practically unchanged and only clay minerals start to dehydrate. At temperatures $500{ }^{\circ} \mathrm{C}$ and above, the kaolinite decomposes to metakaolinite- and mullite-type alumosilicate mass. Nevertheless, at these temperatures $\left(\sim 400-500{ }^{\circ} \mathrm{C}\right)$ various sulfur compounds (mainly in the form of pyrite and/or marcasite $\mathrm{FeS}_{2}$ ) start to decompose. The sulfur released by pyrite decomposition and the free $\mathrm{CaO}$ released from partial pyrolysis of calcium carbonate react and form a CaS-type phase, whereas the uncompleted decomposition process of pyrite may produce mackinawite $[\mathrm{FeS}]$ type phases.

At the final step of retorting the temperatures of $900-1000{ }^{\circ} \mathrm{C}$ are applied to burn off the organic matter/coke in solid residue. In this process a slaglike material forms, which consists of amorphous glass phase at the expense of the decomposition and melting of alumosilicate minerals (clays) and newformed Ca-silicates - melilite, cement minerals like belite $[\beta$-C2S], merwinite, etc. that form in reactions between free $\mathrm{Ca}$ and $\mathrm{Mg}$ oxides derived from the decomposition of dolomite (decomposition starts at $\sim 860{ }^{\circ} \mathrm{C}$ ) and calcite (decomposition at $\sim 950{ }^{\circ} \mathrm{C}$ ) with alumosilicates (mainly clay minerals and K-feldspar) and less quartz. Moreover, mineral composition of slag lumps found in semi-coke indicates that temperatures as high as $1400-1500{ }^{\circ} \mathrm{C}$ can occur locally during this stage.

\section{Hydration and diagenesis of semi-coke sediments}

Mineral phases formed in the oil shale retorting/semi-coking process are unstable in atmospheric conditions. Deposition of spent shale on waste dumps initiates transformation, predominantly hydration and carbonation, of minerals. The dominant hydration product of semi-coke is secondary ettringite $-\mathrm{Ca}_{6} \mathrm{Al}_{2}\left(\mathrm{SO}_{4}\right)_{3}(\mathrm{OH})_{12} \cdot 26 \mathrm{H}_{2} \mathrm{O}$.

It is important to note that in semi-coke waste heaps ettringite acts as a cement mineral, and by binding waste particles forms a monolithic deposit, 
therefore stabilizing semi-coke dumps geotechnically. It has also been shown that ettringite formation controls the activity of several contaminants, both cations and oxoanions and is concomitant with the reduction of leachate trace elements [19].

The formation of ettringite occurs as a result of the reaction of $\mathrm{CaO}$, formed at thermal decomposition of limestone/dolomite, with Al-compounds derived from partial decomposition of clay minerals and/or feldspars, and with dissolved sulfur compounds and/or CaS (oldhamite), emitted in the combustion process of organic matter or decomposition product of pyrite and carbonate phases, respectively. The precipitation kinetics of ettringite is rapid. In laboratory experiments the nucleation of ettringite crystallites is fast, and the white ettringite precipitate appears immediately after mixing of $\mathrm{CaO}$ and $\mathrm{Al}_{2}\left(\mathrm{SO}_{4}\right)_{3}$ solutions [20]. However, ettringite formation in semicoke deposits under atmospheric conditions is much slower, and the ettringite formation occurs over two-three weeks. Although nucleation can be assumed to begin in a matter of hours, the crystalline ettringite appearance takes days or weeks. The controlling factors of ettringite precipitation are concentrations and activities of dissolved $\mathrm{Ca}^{2+}, \mathrm{Al}^{3+}$ and $\mathrm{SO}_{4}{ }^{2-}$ ions, which depend on ionic strength of semi-coke pore-water. In plateau sediments consisting of oil shale combustion ash the ettringite formation is limited by the sulfate deficiency [21], whereas in semi-coke deposits the controlling factor seems to be the availability of $\mathrm{CaO}$ and/or $\mathrm{Ca}(\mathrm{OH})_{2}$. In semi-coke sediments it is probable that at high sulfate activity all of the free $\mathrm{Ca}$ will be bound to form ettringite.

The crucial factor for formation and stability of ettringite is $\mathrm{pH}$. In alkali and sulfate-rich solutions ettringite is the most stable phase of the $\mathrm{Ca}(\mathrm{OH})_{2}-$ $\mathrm{Al}_{2}\left(\mathrm{SO}_{4}\right)_{3}-\mathrm{H}_{2} \mathrm{O}$ system. Ettringite is stable at $\mathrm{pH}$ values $>10.7$, at lower $\mathrm{pH}$ values ettringite dissolves incongruently to gypsum, (amorphous) Alhydroxide and $\mathrm{Ca}$-aluminate type phases. At high $\mathrm{pH}$ values one of the solubility products is portlandite [20]. However, at high $\mathrm{CO}_{2}$ partial pressure and relatively low $\mathrm{pH}$ level, ettringite decomposes into sulfates and aragonite $\left[\mathrm{CaCO}_{3}\right]$ with vaterite $\left[\gamma-\mathrm{CaCO}_{3}\right]$ as an intermediate phase [22]. Moreover, decomposition of ettringite (decomposition rate) is also influenced by temperature and the content of different compounds found in deposit. At high temperatures monosulfoaluminate $\left[\mathrm{Ca}_{4} \mathrm{Al}_{2}\left(\mathrm{SO}_{4}\right)(\mathrm{OH})_{12} \cdot 6 \mathrm{H}_{2} \mathrm{O}\right]$ phase is stable over ettringite, whereas according to Pajares et al., [23] ettringite solubility is inhibited in the presence of $\mathrm{CaCO}_{3}$ (calcite) and cement clinkerminerals: belite $[\mathrm{C} 2 \mathrm{~S}]$, alite $[\mathrm{C} 3 \mathrm{~S}]$ etc. Those phases have the capacity to buffer solution $\mathrm{pH}$ to the critical levels for ettringite stability. When the $\mathrm{Ca}(\mathrm{OH})_{2}-\mathrm{Al}_{2}\left(\mathrm{SO}_{4}\right)_{3}-\mathrm{H}_{2} \mathrm{O}$ system is open to atmospheric $\mathrm{CO}_{2}$, under alkaline $\mathrm{pH}$ conditions its solution approaches saturation of carbonate mineral phases such as calcite and aragonite. However, slow precipitation kinetics of calcite associated with slow transport of $\mathrm{CO}_{2}$ into the waste materials delays its formation relative to ettringite and gypsum. Moreover, high $\mathrm{SO}_{4}{ }^{2-}$ activity favors the formation of gypsum over calcite [24]. This has also been 
observed from field studies of ettringite-bearing, weathered, flue-gas desulfurization (FGD) materials. In these alkaline materials calcite did not appear until 240 days of weathering [24]. However, elevated $\mathrm{CO}_{2}$ partial pressures due to decomposition of organic matter in subsurface soil horizons or prolonged leaching of $\mathrm{SO}_{4}{ }^{2-}$ from waste materials should favor the formation of calcite.

The most prominent difference of the semi-coke compared to oil shale combustion ash sediments [e. g. 25] is the absence of portlandite $\left[\mathrm{Ca}(\mathrm{OH})_{2}\right]$ and very low content of hydrocalumite $\left[\mathrm{Ca}_{2} \mathrm{Al}(\mathrm{OH})_{7} \cdot 3 \mathrm{H}_{2} \mathrm{O}\right]-$ common constituents in ash sediments. However, high $\mathrm{pH}$ (12-13) of initial semi-coke leachates suggests the presence of some amount of portlandite in semi-coke, but its quantity remains probably below $1 \%$ that is under the detection limit of X-ray diffraction analyses. The presence of small amount of portlandite $(\sim 0.6 \%)$ in semi-coke was confirmed by titration experiment where semicoke leachate $\mathrm{pH}$ was measured at different sediment-water ratios. The $\mathrm{pH}$ value in oversaturated solution compared to portlandite is 12.4 at $25{ }^{\circ} \mathrm{C}$ and the portlandite content in semi-coke matter was found from saturationundersaturation equilibrium. The restricted occurrence of portlandite would suggest also limited carbonation of semi-coke sediments. However, the SEM studies show the presence of authigenic calcite aggregates in semi-coke sediments. Preconditions for carbonation process are dissolution of ettringite and the reaction with atmospheric carbon dioxide $\left(\mathrm{CO}_{2}\right)$ to form significantly stronger and less soluble calcium carbonate phase calcite, aragonite or possibly vaterite $\left(\gamma-\mathrm{CaCO}_{3}\right)$. For carbonation to occur, the presence of water is essential, since it requires dissolution of $\mathrm{CO}_{2}$.

The inner structure of semi-coke waste heaps exhibits considerable variation, which is probably due to both physical and chemical separation during deposition of the sediment and due to different diagenetic/hydration processes. The surface layers of the semi-coke waste heaps are under a constant influence of percolating precipitation water, which considerably lowers the $\mathrm{pH}$ of porewater in the upper layers and thus initiates, in the first place, the dissolution of ettringite. The studied sections indicate that, although not well expressed in all sequences, the ettringite dissolution profile can be traced to several meters depth. A remarkably higher content of calcite and dolomite in samples from drillcore P1 is probably location-specific. The site is located on the foot of the semi-coke waste heap (Fig. 3), and the most feasible explanation is gravitational separation during dumping of the material. The material deposited on the studied area was dumped in large mounds. By dumping, the waste material fractionates gravitationally with largest particles transported onto the foot area of the mound [26]. The largest particles in semi-coke are usually partially decomposed harder lumps of oil shale and especially carbonate rocks, which are also reflected in the mineral composition of the sediment. 


\section{Summary and conclusions}

This study of the semi-coke mineralogy and its sediments shows that:

the predominant phases in a fresh unhydrated semi-coke are calcite, dolomite, quartz, K-feldspar and clay minerals (mostly illite). There are also phases like melilite, periclase, belite, merwinite, formed by partial thermal decomposition and subsequent reactions of the initial minerals. It is important to note that the CaS phase oldhamite together with traces of FeS mineral mackinawite were identified in fresh semi-coke. The composition of semi-coke reflects the changes of mineral matter during the retorting process. During the main phase of retorting at temperatures $\sim 400-520^{\circ} \mathrm{C}$ only dehydration and partial transformation of clay minerals as well as decomposition of sulfur compounds (pyrite, marcasite) occur. At the final step of retorting during short heating to temperatures $900-1000{ }^{\circ} \mathrm{C}$ a slaglike material forms, which consists of amorphous and Ca-silicate phases.

During deposition and hydration the composition of semi-coke changes, unstable phases (e.g. oldhamite) disappear, and they are replaced by a considerable amount of Ca-Al-sulfate - ettringite. Ettringite precipitation and stability are controlled by dissolved $\mathrm{Ca}^{2+}, \mathrm{Al}^{3+}$ and $\mathrm{SO}_{4}{ }^{2-}$ ions from dissolution of unstable thermal decomposition products. Important parameter for ettringite formation and stability is $\mathrm{pH}$ as ettringite is stable at $\mathrm{pH}>10.7$ below what ettringite dissolves incongruently to gypsum, (amorphous) Al-hydroxide and Ca-aluminate type phases.

The composition of semi-coke in heaps of oil shale retort waste is relatively uniform. The ettringite content increases slightly in the whole range of drillcore sampling points both vertically and horizontally. The inner structure of semi-coke waste heaps exhibits variation probably due to both physical and chemical separation during deposition of the sediment and to different diagenetic/hydration processes. Surface layers are leached by continuous rainfall, which lowers $\mathrm{pH}$ of porewater in the upper layers and thus initiates dissolution of ettringite. Elevated content of carbonates and, respectively, lower amounts of terrigenous material at the foot of the waste mound can be explained by gravitational fractionation during dumping of the material, with large and hard partially decomposed limestone particles transported onto the foot area of the mound. That is also reflected in the mineral composition of the sediment.

\section{Acknowledgements}

We are grateful to AS Viru Keemia Grupp and to IPT Projektijuhtimine OÜ for their support during the fieldwork. The authors are grateful to Dr. François Marini for critical reviewing the manuscript and for useful remarks. 


\section{REFERENCES}

1. Teedumäe, A., Raukas, A. The possibility of integrating sustainability into legal framework for use of oil shale reserves // Oil Shale. 2006. Vol 23, No. 2. P. 119-124.

2. Golubev, N. Solid heat carrier technology for oil shale retorting // Oil Shale. 2003. Vol. 20, No. 3S. P. 324-332.

3. Kahru, A., Põllumaa, L. Environmental hazard of the waste streams of Estonian oil shale industry: an ecotoxicological review // Oil Shale. 2006. Vol. 23, No. 1. P. 53-93.

4. Veski, $R$. The volumes of spent shale from Estonian oil-shale processing units in 1921-2002 // Oil Shale. 2005. Vol. 22, No. 3. P. 345-357.

5. Kuusik, R., Martins, A., Pihu, T., Pesur, A., Kaljuvee, T., Prikk, A., Trikkel, A., Arro, $H$. Fluidized-bed combustion of oil shale retorting solid waste// Oil Shale. 2004. Vol. 21, No. 3. P. 237-248.

6. Kattai, V., Saadre, T., Savitski, L. Estonian oil shale: geology, resource, mining conditions. Tallinn: Geological Survey of Estonia, 2000. 226 pp. [in Estonian].

7. Ots, A. Oil shale combustion technology // Oil Shale. 2004. Vol. 21, No 2. P. 149-160.

8. Bauert, H., Kattai, V. Kukersite oil shale // Geology and Mineral Resources of Estonia / A. Raukas, A. Teedumae (eds.). Tallinn: Estonian Academy Publishers, 1997. P. 313-327.

9. Koel, M. Estonian oil shale// Oil Shale Extra, 1999. Available at: http://www.kirj.ee/oilshale/Est-OS.htm.

10. Lille, $\ddot{U}$., Heinmaa, I., Mü̈̈risepp, A. M., Pehk, T. Investigation of kukersite structure using NMR and oxidative cleavage: On the nature of phenolc precursors in the kerogen of Estonian kukersite // Oil Shale. 2002. Vol. 19, No. 2. P. 101-116.

11. Gavrilova, O., Randla, T., Vallner, L., Strandberg, M., Vilu, R. Life Cycle Analysis of the Estonian Oil Shale Industry. Estoninan Fund for Nature, Tallinn University of Technology, Tallinn, 2005. 145 pp. Manuscript.

12. Utsal, $K$. Comprehensive investigation of oil shale material composition by X-ray diffrection method// Oil Shale. 1984. Vol. 1, No. 1. P. 69-80 [in Russian].

13. Kann, J., Elenurm, A., Rohtla, I., Golubev, N., Kaidalov, A., Kindorkin, B. About thermal low-temperature processing of oil shale by solid heat carrier method // Oil Shale. 2004. Vol. 22, No. 3. P. 195-203.

14. Soone, J., Doilov, S. Sustainable utilization of oil shale resources and comprasion of contemporary technologies used for oil shale processing // Oil Shale. 2003. Vol. 20, No. 3S. P. 311-323.

15. Taylor, J. C. Computer programs for standardless quantitative analysis of minerals using the full powder diffraction profile // Powder Diffraction. 1991. Vol. 6. P. 2-9.

16. Ward, C. R., Taylor, J. C., Matulis, C. E., Dale, L. S. Quantification of mineral matter in the Argonne Premium Coals using interactive Rietveld-based X-ray diffraction // International Journal of Coal Geology. 2001. Vol. 46. P. 67-82.

17. Dollase, W. A. Correction of intensities for preferred orientation in powder X-ray diffractometry - application of the March model // J. Appl. Crystallogr. 1986. Vol. 19. P. 267-272. 
18. Kuusik, R., Uibu, M., Kirsimäe, K. Characterization of oil shale ashes formed at industrial-scale CFBC boilers // Oil Shale. 2005. Vol. 22, No. 4S. P. 407-419.

19. Fowler, R. K., Traina, S. J., Bigham, J. M., Soto, U. I. Solution chemistry and mineralogy of clean coal technology by-products in a long term equilibration study // Agronomy Abstracts. Madison: American Society of Agronomy, WI, USA, 1993. Vol. 30. P. 361.

20. Myneni, S. C. B., Traina, S. J., Logan, T. L. Ettringite solubility and geochemistry of the $\mathrm{Ca}(\mathrm{OH})_{2}-\mathrm{Al}_{2}\left(\mathrm{SO}_{4}\right)_{3}-\mathrm{H}_{2} \mathrm{O}$ system at 1 atm pressure and 298 K // Chemical Geology. 1998. Vol. 148. P. 1-19.

21. Puura, E. Oil Shale Ash from Thermal Power Plants in Estonia: Its Disposal and Possible Uses // MSc. Thesis. Manchester: Department of Enviromental Biology, Manchester University, 1992. 142 pp.

22. Nishikawa, T., Suzuki, K., Ito, S., Sato, K., Takebe, T. Decomposition of synthetic ettringite by carbonation// Cement and Concrete Research. 1992. Vol. 22. P. 6-14.

23. Pajares, I., Martínez-Ramírez, S., Blanco-Varela, M. T. Evolution of ettringite in presence of carbonate and silicate ions // Cement and Concrete Composite. 2003. Vol. 25. P. 861-865.

24. Doner, H. E., Lynn, W. C. Carbonate, halide, sulfate and sulfide minerals // Minerals in Soil Environments / J. B. Dixon, S. B. Weed (eds.). SSSA Book Series, 1989. Vol. 1. P. 279-324.

25. Kespre, T. Mineralogy of the oil-shale ash deposits at Eesti Thermal Power Plant // MSc. Thesis. Tartu: University of Tartu, Institute of Geology, 2004. 45 pp. [in Estonian].

26. Puura, E., Pihlak, A. Oxidation of dictyonema shale in Maardu mining waste dumps // Oil Shale. 1998. Vol. 15, No. 3. P. 239-267.

Received January 15, 2007 